\title{
Chromophorylation of a Novel Cyanobacteriochrome GAF Domain from Spirulina and Its Response to Copper Ions
}

\author{
Su-Dan Jiang ${ }^{1}$, Yi sheng ${ }^{1}$, Xian-Jun Wu ${ }^{1,2,3 *}$, Yong-Li Zhu ${ }^{1,2,3}$, and Ping-Ping Li ${ }^{1,2,3 *}$ \\ ${ }^{1}$ College of Biology and the Environment, Nanjing Forestry University, Nanjing 210037, P.R. China \\ ${ }^{2}$ Collaborative Innovation Center of Sustainable Forestry in Southern China of Jiangsu Province, Nanjing Forestry \\ University, Nanjing 210037, P.R. China \\ ${ }^{3}$ National Positioning Observation Station of Hung-tse Lake Wetland Ecosystem in Jiangsu Province, Hongze, \\ Jiangsu 223100, P.R. China
}

\begin{abstract}
Cyanobacteriochromes (CBCRs) are phytochrome-related photoreceptor proteins in cyanobacteria and cover a wide spectral range from ultraviolet to far-red. A single GAF domain that they contain can bind bilin(s) autocatalytically via heterologous recombination and then fluoresce, with potential applications as biomarkers and biosensors. Here, we report that a novel red/green CBCR GAF domain, SPI1085g2 from Spirulina subsalsa, covalently binds both phycocyanobilin (PCB) and phycoerythrobilin (PEB). The PCB-binding GAF domain exhibited canonical red/green photoconversion with weak fluorescence emission. However, the PEB-binding GAF domain, SPI 1085g2-PEB, exhibited an intense orange fluorescence $\left(\lambda_{\text {abs. } \max }=520 \mathrm{~nm}, \lambda_{\text {fluor. } \max }=555 \mathrm{~nm}\right)$, with a fluorescence quantum yield close to 1.0. The fluorescence of SPI1085g2-PEB was selectively and instantaneously quenched by copper ions in a concentration-dependent manner and exhibited reversibility upon treatment with the metal chelator EDTA. This study identified a novel PEB-binding cyanobacteriochrome-based fluorescent protein with the highest quantum yield reported to date and suggests its potential as a biosensor for the rapid detection of copper ions.
\end{abstract}

Keywords: Cyanobacteriochrome, phycoerythrobilin, fluorescence quantum yield, biosensor, copper ions, fluorescence quenching

Received: September 25, 2020 Accepted: November 10, 2020

First published online: November 14,2020

${ }^{*}$ Corresponding authors X. Wu

Phone: +86-158-5052-0507

E-mail: xjwu@njfu.edu.cn

P. Li

Phone: +86-25-8542-7210

E-mail: ppli@njfu.edu.cn

Supplementary data for this paper are available on-line only at http://jmb.or.kr.

pISSN $1017-7825$ eISSN 1738-8872

Copyright(C) 2021 by The Korean Society for Microbiology and Biotechnology

\section{Introduction}

Cyanobacteriochromes (CBCRs) are distantly phytochrome-related photoreceptor biliproteins that contain several GAF (cGMP-specific phosphodiesterase/adenylate cyclase/FhlA) domains that act as sensory modules in cyanobacteria [1]. A single GAF domain can bind covalently linear tetrapyrroles and serve as a chromophore. Tetrapyrrole-binding proteins have great spectral diversity and can sense a wide range of wavelengths, from the near-ultraviolet to the near-infrared [2-7]. Most CBCRs utilize phycocyanobilin (PCB) tetrapyrrole as a chromophore and show reversible photoconversion, which is triggered by $Z / E$ isomerization of the $\mathrm{C} 15-\mathrm{C} 16$ double bond between the $\mathrm{C}$ and $\mathrm{D}$ rings of the chromophore $[2,5,8]$. Among PCB-binding CBCRs, red/green CBCR GAF domains that reversibly photoconvert between the red light-absorbing (Pr) thermostable form with $15 Z$-PCB and the green light-absorbing $(\mathrm{Pg})$ metastable form with 15E-PCB have been well characterized [9-12]. Recently, some red/green CBCR GAF domains have been shown to covalently bind not only PCB but also biliverdin (BV), extending their spectral range towards longer wavelengths [13]. Further, some red/green CBCR GAF domains can even heterogeneously bind phycoerythrobilin (PEB) and, in terms of fluorescence brightness, exhibit a much higher fluorescence quantum yield than the PCB- and/or BV-binding GAF domains [14]. PEBbinding GAF domains, despite their blue-shifted spectrum, are appropriate for use as platforms for fluorescent protein-based biosensors due to their intense fluorescence emission.

Fluorescent proteins show fluorescence quenching by specific metal ions, and can be used in metal biosensing applications [15-17]. The fluorescence intensity of fluorescent proteins may be reduced, depending on the specific transition metal environment, i.e., the concentration of metal ions. Several fluorescent proteins, including green fluorescent proteins (GFPs), Discosoma sp. red fluorescent protein (DsRed), and flavin-binding fluorescent proteins (FbFPs), have been investigated for metal-induced fluorescence quenching and have been suggested as optical biosensors for metal quantitation [18-20]. However, few biosensors based on biliproteins such as CBCRs with intense fluorescence emission are available for the detection of metal ions.

In this article, we identified a novel red/green CBCR GAF domain, SPI1085g2 from Spirulina subsalsa, that covalently binds not only PCB but also PEB. PEB-binding SPI1085g2 exhibited an intense orange fluorescence 
with the highest fluorescence quantum yield reported to date. In addition, we confirmed the selective response of SPI1085g2-PEB fluorescence to copper ions in a concentration-dependent manner, indicating the potential of the red/green CBCR GAF domains for development as a biosensor for copper detection.

\section{Materials and Methods \\ Cloning and Plasmid Construction}

The SPI1085g2 (encoding the apoprotein SPI1085g2) gene was amplified from S. subsalsa FACHB351 genomic DNA by polymerase chain reaction (PCR) using Pfu DNA polymerase (Tiangen Biotech, China). The sequence used to design the primers was obtained from the GenBank nucleotide sequence database (Accession No. WP_017306776). The primers used to amplify the SPI1085g2 gene were as follows: forward primer, $5^{\prime}$-CTA GAG CTC TTC CCT CGA AAT TGA GCA GAT TTT CC-3'; reverse primer, 5'-GTT AAG CTT AGC GGG TTT TGG CTA ATA ATT CGG C-3'. The SPI1085g2 product was digested with the restriction enzymes SacI and HindIII and inserted into the plasmid pETDuet-SPI1085g3-ho1::pcyA [21], instead of SPI1085g3 to obtain the plasmid pETDuet-SPI1085g2-ho1::pcyA. The pebS gene (encoding PebS), flanked by the restriction enzymes BglII and XhoI, was synthesized by GenScript (China) based on the sequence from the GenBank nucleotide sequence database (Accession No. WP017303809). The pebS gene was then inserted into BglII- and XhoI-digested pETDuetSPI1085g2-ho1-pcyA instead of pcyA, yielding the plasmid pETDuet-SPI1085g2-ho1::pebS. All the primers were synthesized by GenScript. The sequence of the gene encoding SPI1085g2 was verified by DNA sequencing. The obtained nucleotide and amino acid sequences of SPI1085g2 from S. subsalsa FACHB351 are shown in Fig. S1.

\section{Protein Expression and Purification}

The constructed plasmids, pETDuet-SPI1085g2-ho1::pcyA and pETDuet-SPI1085g2-ho1::pebS, were separately transformed into Escherichia coli BL21(DE3). The transformed cells were cultured at $37^{\circ} \mathrm{C}$ in $100 \mathrm{ml}$ Luria-Bertani (LB) medium supplemented with ampicillin $\left(20 \mu \mathrm{g} \cdot \mathrm{ml}^{-1}\right)$. The cells were kept in an ice bath for $30 \mathrm{~min}$ after the $\mathrm{OD}_{600}$ reached 0.5 . After induction with isopropyl $\beta$-D-thiogalactoside $(1 \mathrm{mM})$ for $12 \mathrm{~h}$ at $18^{\circ} \mathrm{C}$, the cells were centrifuged at $12,000 \times g$ for $5 \mathrm{~min}$ at $4^{\circ} \mathrm{C}$. The cell pellet was resuspended in $5 \mathrm{ml}$ of ice-cold potassium phosphate buffer (KPB, $20 \mathrm{mM}, \mathrm{pH} 7.2$ ) containing $0.5 \mathrm{M} \mathrm{NaCl}$ and disrupted by sonication for $5 \mathrm{~min}$ at $200 \mathrm{~W}$ (JY92-IIN, Scientz Biotechnology, China). The suspension was centrifuged at $12,000 \times g$ for $15 \mathrm{~min}$ at $4^{\circ} \mathrm{C}$, and the supernatant was purified via $\mathrm{Ni}^{2+}$-affinity chromatography on chelating Sepharose (Amersham Biosciences, Sweden) equilibrated with $\mathrm{KPB}$-containing $0.5 \mathrm{M} \mathrm{NaCl}$. The bound proteins remaining on the column were eluted with $1 \mathrm{ml}$ of saline KPB containing $0.5 \mathrm{M}$ imidazole. After collection, the protein sample was dialyzed twice against saline $\mathrm{KPB}[22]$.

\section{SDS-PAGE and Zn-Induced Fluorescence Assay}

Protein samples (50 $\mu \mathrm{l}$ each) were analyzed by polyacrylamide gel electrophoresis (PAGE) in the presence of SDS. The SDS-PAGE gel was composed of a $10 \%$ resolving gel and a $5 \%$ stacking gel. The samples were boiled with $2 \times$ SDS sample buffer containing $30 \mathrm{mM} \beta$-mercaptoethanol for $5 \mathrm{~min}$. The purified proteins were visualized by staining with Coomassie blue, and the bilins in the samples were detected by $\mathrm{Zn}^{2+}$-induced fluorescence. Fluorescence was visualized through a 630-nm filter upon excitation at $530 \mathrm{~nm}$ (GenoSens1850, China).

\section{Spectral Analysis}

All experiments were conducted at room temperature. The absorbance spectra were obtained on a Perkin Elmer Lambda 365 spectrophotometer. After denaturing the proteins in $8 \mathrm{M}$ urea at $\mathrm{pH} 2.0$ in the dark, extinction coefficients were determined by the absorption at: $662 \mathrm{~nm}\left(\varepsilon=35,500 \mathrm{M}^{-1} \mathrm{~cm}^{-1}\right)$ for PCB; $550 \mathrm{~nm}\left(\varepsilon=42,800 \mathrm{M}^{-1}\right.$ $\mathrm{cm}^{-1}$ ) for PEB [23]. Red light was provided by a cold fiber optic light source with a $150 \mathrm{~W}$ halogen lamp (Bocheng, Nanjing, China) through a bandpass filter with a peak wavelength of $653 \mathrm{~nm}$ and a 20 -nm half-bandwidth (Rayan, China), while green light was provided by a light source with a peak wavelength of $535 \mathrm{~nm}$ and a 40 -nm halfbandwidth (Rayan). The light intensity at the sample plane for photoconversion was $15 \mu \mathrm{mol} \mathrm{m}^{-2} \mathrm{~s}^{-1}$. Samples were irradiated for $3 \mathrm{~min}$ for photoconversion. Fluorescence spectra were recorded with a model LS 55 spectrofluorometer (Perkin Elmer, USA). The fluorescence quantum yields were determined in KPB (pH 7.2) using the known $\Phi \mathrm{F}=0.98$ of the biosynthetically obtained $\mathrm{CpcB}(\mathrm{C}-84)$-PEB as standards [23]. The biliprotein concentrations were calculated using the Beer-Lambert Law $(\mathrm{A}=\varepsilon \mathrm{lc})$.

\section{Response Analysis to Heavy Metal}

The effect of heavy metal ions on the fluorescence emission of SPI1085g2-PEB was determined by adding various metal ions $\left(\mathrm{CuSO}_{4}, \mathrm{NiCl}_{2}, \mathrm{MnCl}_{2}, \mathrm{ZnSO}_{4}, \mathrm{CrCl}_{3}, \mathrm{CoCl}_{2}, \mathrm{PbCl}_{2}, \mathrm{CdCl}_{2}\right)$ at a final concentration of $40 \mu \mathrm{M}$ to $0.25 \mu \mathrm{M}$ biliprotein in $\mathrm{KPB}$-containing $0.5 \mathrm{M} \mathrm{NaCl}$. After $30 \mathrm{~min}$ of incubation at $25^{\circ} \mathrm{C}$, the samples were excited at $500 \mathrm{~nm}$, and the emitted fluorescence was measured at $555 \mathrm{~nm}$ on a Perkin Elmer LS-55 spectrofluorometer. The fluorescence intensities were measured at $30 \mathrm{sec}$ intervals for $30 \mathrm{~min}$ to investigate the response time.

To further investigate the effect of $\mathrm{Cu}^{2+}$ on the fluorescence of SPI1085g2-PEB, $10 \mu \mathrm{lof} \mathrm{Cu}^{2+}$ solution at varying concentrations $(0 \sim 5 \mathrm{mM}$ ) was added to $490 \mu \mathrm{l}$ of $0.25 \mu \mathrm{M}$ biliprotein (in KPB-containing $0.5 \mathrm{M} \mathrm{NaCl}, \mathrm{pH} 7.2$ ). After $30 \mathrm{~min}$ of incubation at $25^{\circ} \mathrm{C}$, the fluorescence at $555 \mathrm{~nm}$ of the samples and the emission spectra were recorded after excitation at $500 \mathrm{~nm}$. To assess the reversibility of the fluorescence quenching of SPI1085g2-PEB, simultaneous addition of different concentrations of EDTA to the biliprotein solution with the addition of $\mathrm{Cu}^{2+}$ was carried out. After $30 \mathrm{~min}$ of incubation at $25^{\circ} \mathrm{C}$, the fluorescence emission spectra of the samples were recorded. All experiments were performed in triplicate at $25^{\circ} \mathrm{C}$. 


\section{Stern-Volmer Plot and Dissociation Constant}

A Stern-Volmer plot was generated for $0.25 \mu \mathrm{M}$ biliprotein with different concentrations of $\mathrm{Cu}^{2+}$ using the equation as follows:

$$
F / F_{0}=1+K_{\mathrm{SV}}[\mathrm{Q}]
$$

Here, $F$ is the measured fluorescence intensity in presence of $\mathrm{Cu}^{2+}, F_{0}$ is the measured fluorescence intensity without $\mathrm{Cu}^{2+},[\mathrm{Q}]$ is the concentration of $\mathrm{Cu}^{2+}$, and $K_{\mathrm{SV}}$ is the Stern-Volmer constant. The curve for $F / F_{0}$ against copper concentration was linearly fitted using the equation, and the slope was determined to give the value of Ksv.

The dissociation constant for copper $\left(K_{\mathrm{d}}\right)$ was calculated by plotting the $\left[\Delta F / \Delta F_{\max }\right]$ against the different concentrations of $\mathrm{Cu}^{2+}$, fitted using the equation as follows [18]:

$$
\Delta F / \Delta F_{\max }=\left(K_{\mathrm{d}}+[\mathrm{P}]+[\mathrm{Cu}]-\sqrt{\left(K_{\mathrm{d}}+[\mathrm{P}]+[\mathrm{Cu}]\right)^{2}-4[\mathrm{P}][\mathrm{Cu}]}\right) / 2[\mathrm{P}],
$$

where $\Delta F$ is the change in measured fluorescence, $\Delta F_{\max }$ is the maximum fluorescence change, $K_{\mathrm{d}}$ is the dissociation constant, $[\mathrm{P}]$ is the protein concentration, and $[\mathrm{Cu}]$ is the concentration of $\mathrm{Cu}^{2+}$. The curve for $\Delta F /$ $\Delta F_{\max }$ against copper concentration was fitted using the equation and $K_{\mathrm{d}}$ was calculated. The strength of interactions between protein and a ligand is inversely related to the value of $K_{\mathrm{d}}$.

\section{Results}

\section{Chromophorylation of SPI1085g2}

SPI1085, a hypothetical signal transduction protein from S. subsalsa, contains four GAF domains, as previously reported, one of which, SPI1085g3, can bind PCB as a chromophore and exhibits a red/dark fluorescence switch [21]. Here, we cloned the DNA segment encoding the second GAF domain of SPI1085 (SPI1085g2, residues 216369) from the S. subsalsa FACHB351 genome (Fig. 1A). To evaluate the bilin-binding capability of SPI1085g2, we first tested the chromophorylation of SPI1085g2 with PCB. SPI1085g2 can covalently bind PCB, as demonstrated by the zinc-dependent fluorescence observed by SDS-PAGE (Fig. 1B), despite exhibiting a low bilin-binding rate, and by spectral and acid denaturation analysis of chromophorylated SPI1085g2 (Fig. 1C). The PCB-binding SPI1085g2 (SPI1085g2-PCB) showed reversible photoconversion between a red-absorbing Pr form at $642 \mathrm{~nm}$ and a green-absorbing Pg form at $535 \mathrm{~nm}$ (Fig. 1C), which was very similar to that of Slr1393g3 from Synechocystis sp. PCC 6803 [10]. We compared the primary sequence of SPI1085g2 with those of Slr1393g3 and another red/green CBCR GAF domain, All2699g3 [24]. Some residues conserved or enriched in typical red/green CBCR GAF domains appear in SPI1085g2 (Fig. S2). Therefore, SPI1085g2 belongs to the canonical red/green CBCRs. Next, chromophorylation with PEB instead of PCB as the chromophore was performed and unexpectedly resulted in highly efficient binding of PEB to SPI1085g2, as indicated by a band with strong zinc-dependent fluorescence (Fig. 1B) and spectral and acid denaturation analysis (Fig. 1D). Furthermore, we failed to obtain chromophorylated SPI1085g2 with BV as demonstrated by the zinc-dependent fluorescence (Fig. 1B). The PEB-binding SPI1085g2 (SPI1085g2-PEB) was not photochromic and exhibited maximum absorption at $520 \mathrm{~nm}$ (Fig. 1D). Although PEB

A

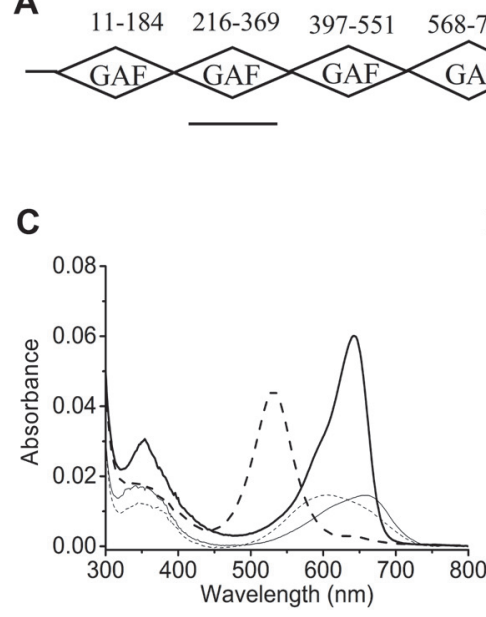

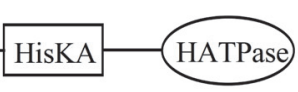

D

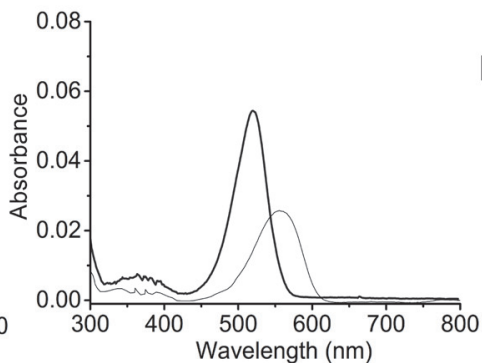

B

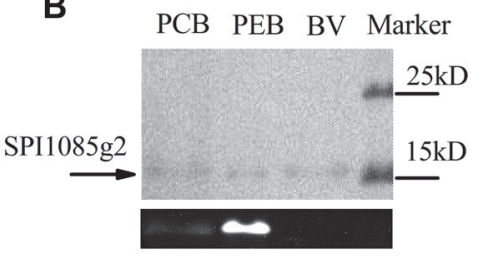

$\mathbf{E}$

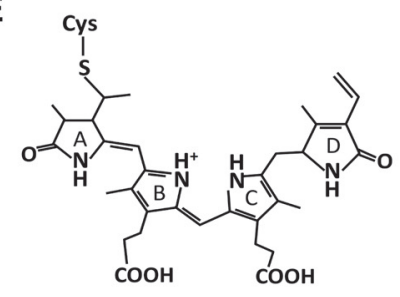

Fig. 1. Domain position and chromophorylation of SPI1085g2. (A) Domain architecture of SPI9445_RS0121085 (SPI1085). GAF: cGMP-specific phosphodiesterase/adenylate cyclase/FhlA; HisKA: histidine kinase acceptor; HATPase: histidine associated ATPase. The underline indicates the SPI1085g2 position. (B) SDS-PAGE analyses of SPI1085g2 chromophorylated with PCB (phycocyanobilin), PEB (phycoerythrobilin) and BV (biliverdin): Coomassie brilliant blue staining (upper gel image) and zinc-induced fluorescence (lower gel image). (C) Absorbance spectra of native (heavy lines) and acid-urea-denatured (thin lines) SPI1085g2-PCB. The dashed lines correspond to the $15 \mathrm{E}$ state obtained after irradiation with $653 / 20$-nm light, and the solid lines correspond to the $15 \mathrm{Z}$ state obtained after irradiation with 530/20-nm light. (D) Absorbance spectra of native (heavy line) and acid-urea-denatured (thin line) SPI1085g2-PEB. (E) The structure of phycoerythrobilin (PEB). 
A

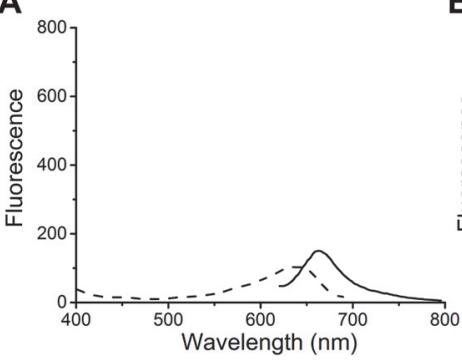

B

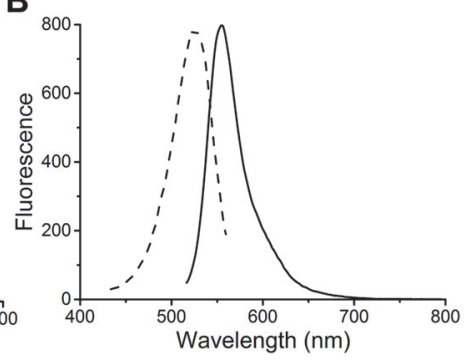

C

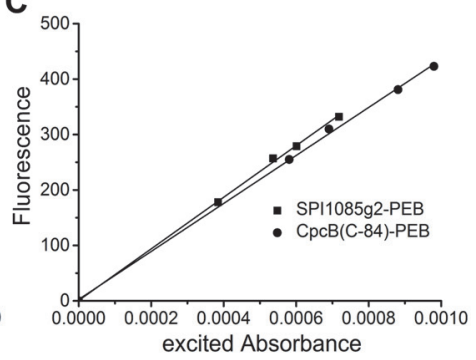

Fig. 2. Fluorescence properties of chromophorylated SPI1085g2. Fluorescence excitation (dashed line) and emission (solid line) spectra of SPI1085g2-PCB, excited at $600 \mathrm{~nm}$ (A), and SPI1085g2-PEB, excited at $480 \mathrm{~nm}$ (B). (C) Plot of fluorescence emission vs. excited absorbance for the dilution series of SPI1085g2-PEB (solid line) and CpcB(C-84)-PEB (dashed line). The slopes of these lines are proportional to the quantum yields for fluorescence.

also covalently binds to Slr1393g3 and All2699g3, they are partially converted into phycourobilin (PUB), which results in a lower yield [14]. The PEB bound to SPI1085g2 was not further modified. Therefore, SPI1085g2-PEB was more similar to the previously reported PEB-binding All2699g1 (All2699g1-PEB) but with a remarkably blueshifted absorption maximum compared to All2699g1 with absorption maximum at $575 \mathrm{~nm}$ [14]. Clearly, the PEBbinding GAF domains could be divided into two categories, Slr1393g3 and All2699g3 with isomerization of PEB to PUB and lower yield, and SPI1085g2 and All2699g1 with non-isomerization and higher yield, respectively. We compared the amino acid sequences of the four GAF domains based on multiple alignment (Fig. S2). SPI1085g2 and All2699g1 showed great differences in some conserved amino acid residues. It was speculated that some identical residues in SPI1085g2 and All2699g1 might play important roles for PEB incorporation.

\section{Intense Fluorescence of SPI1085g2-PEB}

To study the fluorescence properties of chromophorylated SPI1085g2, fluorescence spectra at room temperature were measured. The results showed that the thermostable forms of SPI1085g2-PCB exhibited only weak fluorescence with an emission maximum at $662 \mathrm{~nm}$ (Fig. 2A), whereas SPI1085g2-PEB exhibited an intense orange fluorescence with an emission maximum at $555 \mathrm{~nm}$ (Fig. 2B). Furthermore, the fluorescence quantum yield of SPI1085g2-PEB was calculated to be very close to 1.0 (Table 1), which was comparable with that of some phycobiliproteins attached to PEB (Fig. 2C) but higher than that of All2699g1 $(\Phi \mathrm{F}=0.55)$ and Cph1 $(\Phi \mathrm{F}=0.72)$ $[14,23,25]$. Moreover, SPI1085g2-PEB also has a larger Stokes shift $(35 \mathrm{~nm})$ than PEB-binding phycobiliproteins All2699g1 and Cph1 for the reduction of scatter interference.

\section{Response of SPI1085g2-PEB to Copper}

To evaluate the potential of SPI1085g2 as a single fluorescent protein biosensor for heavy metal detection, we conducted spectroscopic studies to investigate whether SPI1085g2-PEB can be fluorescently quenched by various heavy metal ions. The results demonstrated that the fluorescence of SPI1085g2-PEB was not substantially affected by the addition of $0.04 \mathrm{mM}$ heavy metal ions, such as $\mathrm{Cd}^{2+}, \mathrm{Co}^{2+}, \mathrm{Cr}^{3+}, \mathrm{Mn}^{2+}, \mathrm{Ni}^{2+}, \mathrm{Pb}^{2+}$, and $\mathrm{Zn}^{2+}$. However, the addition of $0.04 \mathrm{mM} \mathrm{Cu}^{2+}$ exerted a maximum fluorescence quenching of approximately $75 \%$ (Fig. 3A). Therefore, among the tested heavy metal ions, only $\mathrm{Cu}^{2+}$ caused selective fluorescence quenching of SPI1085g2-PEB. Once the $\mathrm{Cu}^{2+}$ was added into SPI1085g2-PEB, the fluorescence intensity at $555 \mathrm{~nm}$ was recorded over time (0-30 min) to investigate the response time of SPI1085g2-PEB toward $\mathrm{Cu}^{2+}$. A large instantaneous decrease in fluorescence intensity was observed within less than $5 \mathrm{sec}$ after adding $\mathrm{Cu}^{2+}$ and the intensity continued to decline slowly over time (Fig. 3B). This indicates that SPI1085g2-PEB exhibited very short response time to $\mathrm{Cu}^{2+}$ and provided an advantage for biosensor applications.

Next, the responding properties of SPI1085g2-PEB for $\mathrm{Cu}^{2+}$ were analyzed by incubating equimolar amounts of purified fluorescent proteins with $0-40 \mu \mathrm{M} \mathrm{Cu}^{2+}$. The fluorescence emission spectrum of SPI1085g2-PEB indicated a reduction of fluorescence intensity with increasing $\mathrm{Cu}^{2+}$ concentration, while the shape of the emission signal and its position did not change (Fig. 4A). To confirm that the fluorescence quenching we observed was reversible, we treated mixtures of $0.25 \mu \mathrm{M}$ SPI1085g2-PEB and $40 \mu \mathrm{M} \mathrm{Cu}^{2+}$ with $0.025-25 \mu \mathrm{M}$ EDTA. We found that the fluorescence emission spectrum of the quenched fluorescent proteins gradually reverted close to the initial state with the increase of the EDTA concentration (Fig. 4B), demonstrating that the quenching effect was

Table 1. Spectral properties of reconstituted biliproteins.

\begin{tabular}{ccccc}
\hline \multirow{2}{*}{ Biliprotein } & \multicolumn{2}{c}{ Absorbance } & \multicolumn{2}{c}{ Fluorescence } \\
\cline { 2 - 5 } & $\lambda_{\max }[\mathrm{nm}]$ & $\varepsilon_{\text {vis }}\left[\mathrm{M}^{-1} \cdot \mathrm{cm}^{-1}\right] \times 10^{-4}$ & $\lambda_{\max }[\mathrm{nm}]$ & $\Phi 5$ \\
\hline SPI1085g2-PEB & 520 & 7.4 & 555 & 0.996 \\
SPI1085g2-PCB & 642 & 8.3 & 662 & 0.007 \\
\hline
\end{tabular}

Spectra were obtained in potassium phosphate buffer $(20 \mathrm{mM}, \mathrm{pH} 7.0)$. Extinction coefficients and fluorescence yields were averages of two independent experiments. 
A

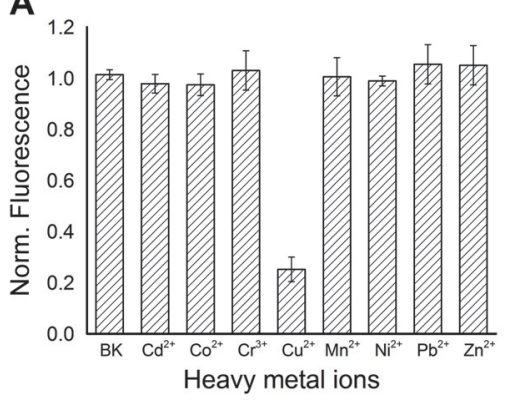

B

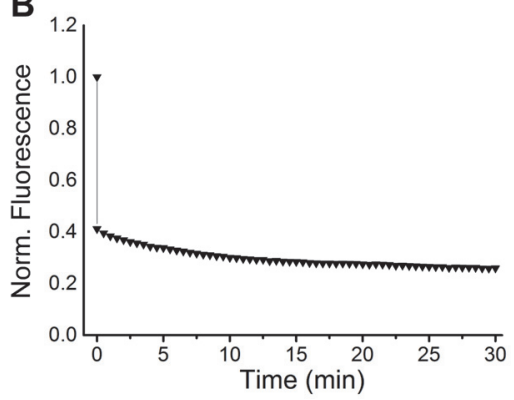

Fig. 3. Selectivity and response time of SPI1085g2-PEB. (A) Effects of different heavy metal ions on the fluorescence emission of SPI1085g2-PEB. (B) Response time of SPI1085g2-PEB in the presence of $\mathrm{Cu}^{2+}$.

due to the presence of the metal ion.

A more detailed analysis of fluorescence quenching as a function of the copper concentration for SPI1085g2PEB was performed over a broad concentration range from 0.01 to $100 \mu \mathrm{M}$. The results showed that the fluorescence intensity decreased with increasing copper concentration (Fig. 4C). Only a 10\% decrease in emission was observed when $1 \mu \mathrm{M} \mathrm{Cu}^{2+}$ was added, yet $\mathrm{Cu}^{2+}$ in the range of 1 to $100 \mu \mathrm{M}$ caused a remarkable decrease of fluorescence intensity. Furthermore, there was a sensitive dose-effect relationship between the fluorescence intensity and the concentration of $\mathrm{Cu}^{2+}$ over a narrower range from 2 to $10 \mu \mathrm{M}$, and a decrease of fluorescence intensity with increasing copper concentration was clearly observed (Fig. 4D). The data were fitted to calculate the dissociation constant of SPI1085g2-PEB. The dissociation constant of the copper binding site on the rAsGFP (recombinant Anemonia sulcata green fluorescent protein) was identified as $14.18 \pm 0.21 \mu \mathrm{M}$, which indicates the affinity of SPI1085g2-PEB for $\mathrm{Cu}^{2+}$. The copper dissociation constant of SPI1085g2-PEB was similar to that of DsRed mutant drFP583 [26], but higher than that of DsRed, GFPdopa, and CreiLOV [18-20].

To exhibit a linear dose-effect relationship, a Stern-Volmer plot was generated at room temperature and a linear plot was obtained with a correlation coefficient of 0.9936 (Fig. $5 \mathrm{~A}$ ). The $K_{\mathrm{SV}}$ value was evaluated as $0.5 \times 10^{5} \mathrm{M}^{-1}$ according to the slope of the fitted straight line. Meanwhile, the dose response curve of $\mathrm{Cu}^{2+}$ exposure $(8-40 \mu \mathrm{M})$ demonstrated excellent linearity with a correlation coefficient of 0.9937 (Fig. 5B). This sensitivity covers the maximum allowed copper concentration in drinking water suggested by the World Health Organization, $(31.15 \mu \mathrm{M})$ [27], above which aqueous solution may pose a threat to human health. These observations indicate that SPI1085g2-PEB has potential as a biosensor to be applied to the detection of copper ions.
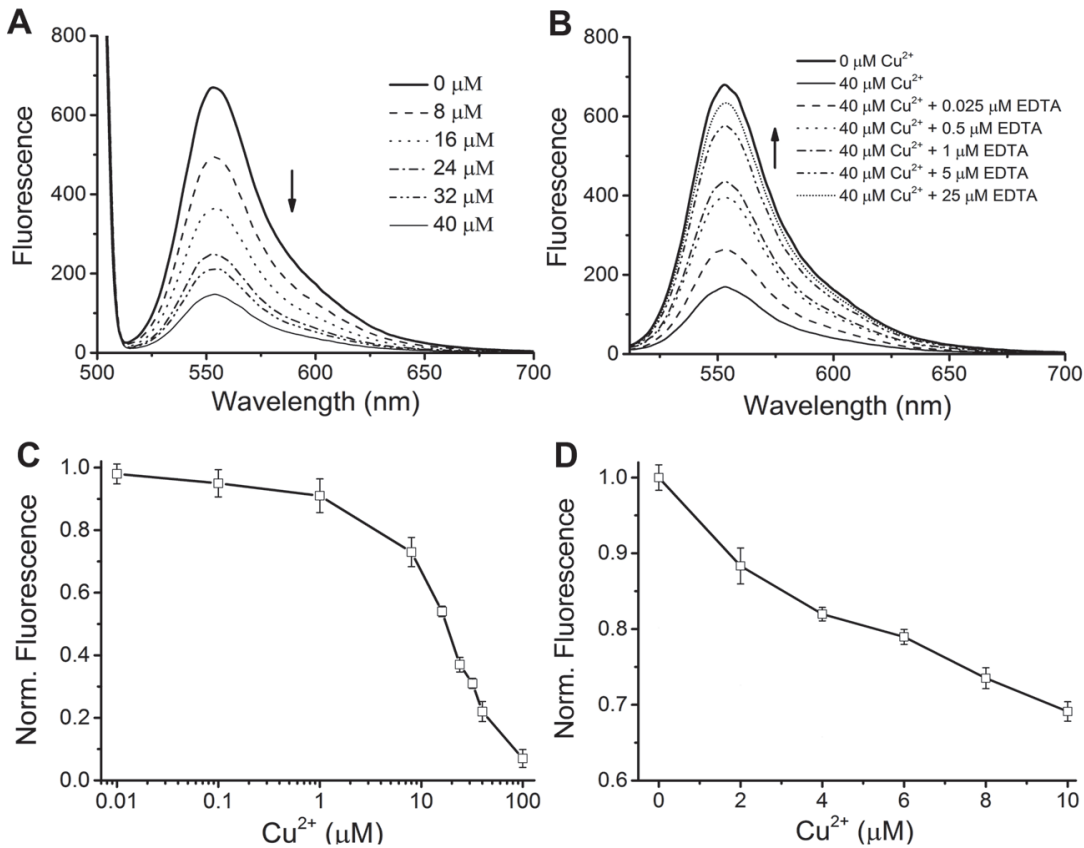

Fig. 4. Response of SPI1085g2-PEB in a concentration-dependent manner. (A) Effect of the presence of different copper concentrations on the fluorescence emission of SPI1085g2-PEB $(0.25 \mu \mathrm{M})$. (B) Reversibility of the $\mathrm{Cu}^{2+}$-mediated quenching of SPI1085g2-PEB. (C) Dose-response curve of SPI1085g2-PEB against $\mathrm{Cu}^{2+}$ in a broad range from 0.01 to $100 \mu \mathrm{M}$. (D) Dose-response curve generated for SPI1085g2-PEB with different concentrations of $\mathrm{Cu}^{2+}$ in a narrower range from 2 to $10 \mu \mathrm{M}$. 

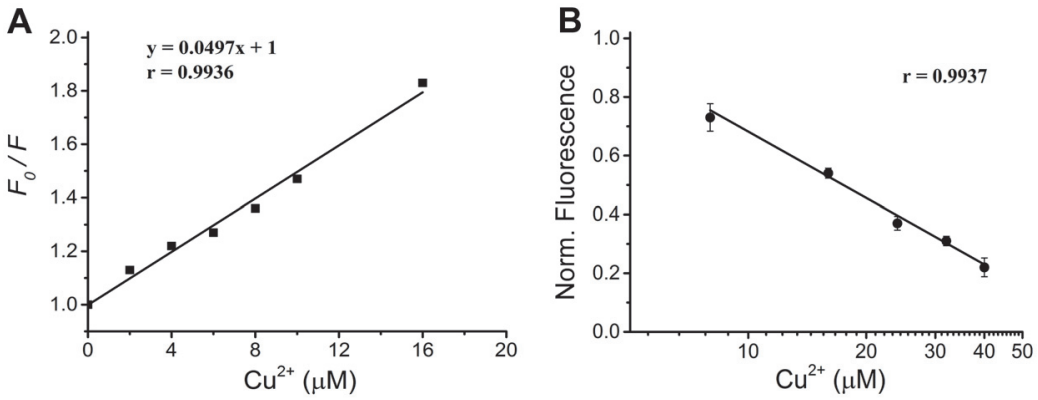

Fig. 5. Linear relationship of SPI1085g2-PEB against $\mathrm{Cu}^{2+}$ in the micromolar range. (A) Stern-Volmer plot generated for SPI1085g2-PEB by the addition of $\mathrm{Cu}^{2+}$ at different concentrations. (B) The plot of the normalized fluorescence intensity of SPI1085g2-PEB vs. $\mathrm{Cu}^{2+}$ concentration $(8-40 \mu \mathrm{M})$. Measurements were repeated 3 times and data were fitted linearly.

\section{Discussion}

CBCRs chromophorylated with different tetrapyrrole chromophores exhibit a great diversity of spectral properties [6]. In the current study, we tested the chromophorylation of a novel red/green CBCR GAF domain of Spirulina, SPI1085g2. This GAF domain binds PCB but cannot bind BV. PEB chromophorylated SPI1085g2 exhibits a fluorescence quantum yield close to 1.0, higher than those of all reported biliproteins. SPI1085g2-PEB was not photochromic with intense orange fluorescence, similar to the previously reported All2699g1-PEB [14]. However, SPI1085g2-PEB exhibited blue-shifted absorption and emission spectra from 575 to $520 \mathrm{~nm}$ in absorption maximum and from 586 to $555 \mathrm{~nm}$ in emission maximum compared with All2699g1-PEB. Moreover, SPI1085g2PEB had a larger Stokes-shift and higher fluorescence quantum yield close to unity than those of All2699g1-PEB. This novel GAF domain expands the spectral variation of biliprotein-based fluorescent constructs and can be used in combination with All2699g1 in flexible applications, such as dual-color labeling and FRET experiments. In terms of both the wavelengths of excitation and emission and fluorescence quantum yield, SPI1085g2-PEB was very similar to $\mathrm{CpcB}-\mathrm{PEB}$, a previously reported orange fluorescing phycobiliprotein [23]. However, the chromophorylation of CBCR GAF domains does not require the involvement of a lyase, facilitating chromophore assembly, compared to that of phycobiliproteins. SPI1085g2-PEB, which has the most intense fluorescence quantum yield reported to date, may be a promising developmental platform for fluorescent biosensor devices.

Direct conversion of fluorescent proteins into a sensor tool for detecting metal ions is currently attracting much interest. We investigated the possibility of using SPI1085g2-PEB as a single biosensor to detect heavy metal ions. The fluorescence of SPI1085g2-PEB can be selectively and instantaneously quenched by $\mathrm{Cu}^{2+}$ in a concentrationdependent manner and recovered almost to the initial fluorescence with the addition of EDTA. SPI1085g2-PEB provided a much faster response time compared to other fluorescent proteins, such as His6GFP (chimeric green fluorescent protein harboring hexahistidine) [28], rAsGFP [29], and iLOV (variant derived through engineering of the light, oxygen or voltage sensing domain) [30], which suggests the potential of SPI1085g2-PEB as a biosensor for rapid detection of copper ions. In this case, the observed fluorescence changes may be due to the interaction between $\mathrm{Cu}^{2+}$ and metal-binding sites, which presumably cause static quenching, as well as that of His6GFP, which bring copper close to the chromophore of the proteins to form a nonfluorescent ground state complex. Consistent with this hypothesis, the emission spectrum of SPI1085g2-PEB did not change in shape or position, but a decrease in intensity was clearly demonstrated. The PEB chromophore unit may be considered as a binding site for $\mathrm{Cu}^{2+}$, because previous studies tend to suggest the tetrapyrrole core as the probable binding site for heavy metal ions [31]. On the other hand, the intramolecular histidine and cysteine residues and the polyhistidine-tag at the Nterminus of SPI1085g2-PEB may also be responsible for binding copper since copper ions prefer to coordinate with nitrogen and thiolate groups typically present in the side chains of histidine and cysteine [32]. In brief, copper ions are bound to either the tetrapyrrole chromophore or the amino-acid residues, or both. This remains to be proved.

The fluorescence reversibility of SPI1085g2-PEB suggests that the continuous and reproducible monitoring of exchangeable copper ions is possible with the protein, which is important for cost-effective application of biosensor materials. The fluorescence recovery of $\mathrm{Cu}^{2+}$-binding SPI1085g2-PEB in the presence of some specific molecules could extend the applications of SPI1085g2-PEB into the detection of mercapto-biomolecules such as homocysteine [33]. SPI1085g2-PEB can be integrated into some immobilized carriers, such as Sol-gel [27], amine-coated glass surfaces [18], and nanocellulose matrices [34], enhancing its stability and facilitating reutilization of the systems. A very small amount of SPI1085g2-PEB is required for the response to copper due to its higher fluorescence quantum yield than those of GFP derivatives [15, 27], iq-FPs [16], DsRed [19], HeRed [35], Dronpa [36], ZsYellow [37], and CreiLOV [20]. Furthermore, a large number of CBCRs have been identified and may provide a set of available libraries for single fluorescent protein biosensors due to their novel photophysical and biochemical properties.

\section{Acknowledgments}

This work was supported by the Natural Science Foundation of Jiangsu Province, China (No. BK20150884), the National Natural Science Foundation of China (No. 41977354 and No. 41471191), China Postdoctoral Science Foundation (No. 14ZR1401500), Jiangsu Planned Projects for Postdoctoral Research Funds (No. 1501020A), and Priority Academic Program Development of Jiangsu Higher Education Institutions (PAPD). 


\section{Conflict of Interest}

The authors have no financial conflicts of interest to declare.

\section{References}

1. Ikeuchi M, Ishizuka T. 2008. Cyanobacteriochromes: a new superfamily of tetrapyrrole-binding photoreceptors in cyanobacteria. Photochem. Photobiol. Sci. 7: 1159-1167.

2. Rockwell NC, Martin SS, Feoktistova K, Lagarias JC. 2011. Diverse two-cysteine photocycles in phytochromes and cyanobacteriochromes. Proc. Natl. Acad. Sci. USA 108: 11854-11859.

3. Rockwell NC, Martin SS, Gulevich AG, Lagarias JC. 2012. Phycoviolobilin formation and spectral tuning in the DXCF cyanobacteriochrome subfamily. Biochemistry 51: 1449-1463.

4. Enomoto G, Hirose Y, Narikawa R, Ikeuchi M. 2012. Thiol-based photocycle of the blue and teal light-sensing cyanobacteriochrome Tlr1999. Biochemistry 51: 3050-3058.

5. Rockwell NC, Martin SS, Lagarias JC. 2012. Red/green cyanobacteriochromes: sensors of color and power. Biochemistry 51: $9667-9677$.

6. Rockwell NC, Martin SS, Lagarias JC. 2016. Identification of cyanobacteriochromes detecting far-red light. Biochemistry 55:3907-3919.

7. Narikawa R, Fushimi K, Ni-Ni-Win, Ikeuchi M. 2015. Red-shifted red/green-type cyanobacteriochrome AM1_1870g3 from the chlorophyll d-bearing cyanobacterium Acaryochloris marina. Biochem. Biophys. Res. Commun. 461: 390-395.

8. Hirose Y, Rockwell NC, Nishiyama K, Narikawa R, Ukaji Y, Inomata K, et al. 2013. Green/red cyanobacteriochromes regulate complementary chromatic acclimation via a protochromic photocycle. Proc. Natl. Acad. Sci. USA 110: 4974-4979.

9. Narikawa R, Fukushima Y, Ishizuka T, Itoh S, Ikeuchi M. 2008. A novel photoactive GAF domain of cyanobacteriochrome AnPixJ that shows reversible green/red photoconversion. J. Mol. Biol. 380: 844-855.

10. Zhang J, Wu XJ, Wang ZB, Chen Y, Wang X, Zhou M, et al. 2010. Fused-gene approach to photoswitchable and fluorescent biliproteins. Angew. Chem. Int. Ed. Engl. 49: 5456-5458.

11. Kim PW, Freer LH, Rockwell NC, Martin SS, Lagarias JC, Larsen DS. 2012. Femtosecond photodynamics of the red/green cyanobacteriochrome NpR6012g4 from Nostoc punctiforme. 1. Forward dynamics. Biochemistry 51: 608-618.

12. Fushimi K, Enomoto G, Ikeuchi M, Narikawa R. 2017. Distinctive properties of dark reversion kinetics between two red/green-type cyanobacteriochromes and their application in the photoregulation of cAMP synthesis. Photochem. Photobiol. 93: 681-691.

13. Fushimi K, Nakajima T, Aono Y, Yamamoto T, Ni-Ni-Win, Ikeuchi M, et al. 2016. Photoconversion and fluorescence properties of a red/green-type cyanobacteriochrome AM1_C0023g2 that binds not only phycocyanobilin but also biliverdin. Front. Microbiol. 7: 588 .

14. Sun YF, Xu JG, Tang K, Miao D, Gärtner W, Scheer H, et al. 2014. Orange fluorescent proteins constructed from cyanobacteriochromes chromophorylated with phycoerythrobilin. Photochem. Photobiol. Sci. 13: 757-763.

15. Richmond TA, Takahashi TT, Shimkhada R, Bernsdorf J. 2000. Engineered metal binding sites on green fluorescence protein. Biochem. Biophys. Res. Commun. 268: 462-465.

16. Yu X, Strub MP, Barnard TJ, Noinaj N, Piszczek G, Buchanan SK, et al. 2014. An engineered palette of metal ion quenchable fluorescent proteins. PLoS One 9: e95808.

17. Bae JE, Kim IJ, Nam KH. 2018. Spectroscopic analysis of the $\mathrm{Cu}^{2+}$-induced fluorescence quenching of fluorescent proteins AmCyan and mOrange2. Mol. Biotechnol. 60: 485-491.

18. Ayyadurai N, Saravanan Prabhu N, Deepankumar K, Lee SG, Jeong HH, Lee CS, et al. 2011. Development of a selective, sensitive, and reversible biosensor by the genetic incorporation of a metal-binding site into green fluorescent protein. Angew. Chem. Int. Ed. Engl. 50: 6534-6537.

19. Sumner JP, Westerberg NM, Stoddard AK, Hurst TK, Cramer M, Thompson RB, et al. 2006. DsRed as a highly sensitive, selective, and reversible fluorescence-based biosensor for both $\mathrm{Cu}^{+}$and $\mathrm{Cu}^{2+}$ ions. Biosens. Bioelectron. 21: 1302-1308.

20. Zou W, Le K, Zastrow ML. 2020. live-cell copper-induced fluorescence quenching of the flavin-binding fluorescent protein CreiLOV. Chembiochem. 21: 1356-1363.

21. Wu XJ, Yang H, Sheng Y, Zhu YL, Li PP. 2018. Fluorescence properties of a novel cyanobacteriochrome GAF domain from Spirulina that exhibits moderate dark reversion. Int. J. Mol. Sci. 19: 2253.

22. Blot N, Wu XJ, Thomas JC, Zhang J, Garczarek L, Böhm S, et al. 2009. Phycourobilin in trichromatic phycocyanin from oceanic cyanobacteria is formed post-translationally by a phycoerythrobilin lyase-isomerase. J. Biol. Chem. 284: 9290-9298.

23. Wu XJ, Chang K, Luo J, Zhou M, Scheer H, Zhao KH. 2013. Modular generation of fluorescent phycobiliproteins. Photochem. Photobiol. Sci. 12: 1036-1040.

24. Chen Y, Zhang J, Luo J, Tu JM, Zeng XL, Xie J, et al. 2012. Photophysical diversity of two novel cyanobacteriochromes with phycocyanobilin chromophores: photochemistry and dark reversion kinetics. FEBS J. 279: 40-54.

25. Mukougawa K, Kanamoto H, Kobayashi T, Yokota A, Kohchi T. 2006. Metabolic engineering to produce phytochromes with phytochromobilin, phycocyanobilin, or phycoerythrobilin chromophore in Escherichia coli. FEBS Lett. 580: 1333-1338.

26. Eli P, Chakrabartty A. 2006. Variants of DsRed fluorescent protein: development of a copper sensor. Protein Sci. 15: $2442-2447$.

27. Fitzgerald DJ. 1998. Safety guidelines for copper in water. Am. J. Clin. Nutr. 67: 1098S-1102S.

28. Isarankura-Na-Ayudhya C, Tantimongcolwat T, Galla HJ, Prachayasittikul V. 2010. Fluorescent protein-based optical biosensor for copper ion quantitation. Biol. Trace Elem. Res. 134: 352-363.

29. Masullo T, Puccio R, Di Pierro M, Tagliavia M, Censi P, Vetri V, et al. 2014. Development of a biosensor for copper detection in aqueous solutions using an Anemonia sulcata recombinant GFP. Appl. Biochem. Biotechnol. 172: 2175-2187.

30. Ravikumar Y, Nadarajan SP, Lee CS, Rhee JK, Yun HD. 2015. A new-generation fluorescent-based metal sensor - iLOV protein. J. Microbiol. Biotechnol. 25: 503-510.

31. Suresh M, Mishra SK, Mishra S, Das A. 2009. The detection of $\mathrm{Hg}^{2+}$ by cyanobacteria in aqueous media. Chem. Commun. 14:2496-2498.

32. Frausto da Silva JJF, Williams RJP. 1991. The Biological Chemistry of Elements. Clarendon Press, Oxford.

33. Puangploy P, Oaew S, Surareungchai W. 2015. Development of fluorescent phycocyanin-Cu ${ }^{2+}$ chemosensor for detection of homocysteine. Int. J. Biosci. Biochem. Bioinform. 5: 241-248.

34. Weishaupt R, Siqueira G, Schubert M, Kämpf MM, Zimmermann T, Maniura-Weber K, et al. 2017. A protein-nanocellulose paper for sensing copper ions at the nano- to micromolar level. Adv. Funct. Mater. 27: 1604291.

35. Rahimi Y, Shrestha S, Banerjee T, Deo SK. 2007. Copper sensing based on the far-red fluorescent protein, HcRed, from Heteractis crispa. Anal. Biochem. 370: 60-67.

36. Kim IJ, Kim S, Park J, Eom I, Kim S, Kim JH, et al. 2016. Crystal structures of Dronpa complexed with quenchable metal ions provide insight into metal biosensor development. FEBS Lett. 590: 2982-2990.

37. Kim IJ, Xu Y, Nam KH. 2020. Spectroscopic and structural analysis of $\mathrm{Cu}^{2+}$-induced fluorescence quenching of ZsYellow. Biosensors (Basel) 10:29. 